\title{
A SEPARATION OF SYSTEMATIC AND TURBULENT MOTIONS IN THE NARROW-LINE REGION OF NGC 4151
}

\author{
HARTMUT SCHULZ and BERTO BOER \\ Astronomical Institute of the Ruhr-University \\ Postfach 102148 \\ D-4630 Bochum 1 \\ Federal Republic of Germany
}

\begin{abstract}
Line profiles from the spatially resolved narrow-line region of NGC 4151 are separated into several bulk-motion components which show velocity dispersions two to three times larger than starburst nuclei with similar luminosity. The components are suggested to arise within the galactic disk and by filaments entrained in or situated along the shells of two superbubbles. The large cone-like $S W$ bubble is able to account for the $S W$ soft-X ray excess and the hole in the NLR disk through which a UV-radiation cone emerges. The bubbles appear to be related to the nuclear radio source.
\end{abstract}

Even though early spectra by Ulrich (1973) clearly revealed the basic kinematical features of the extended narrow-line region (NLR) in NGC 4151, the debate on the fundamental shape of its velocity field has not yet been settled. We revisit this question utilizing 30 long-slit image-tube and two CCD spectra (see Schulz 1987). As an aid for the interpretation we computed a large variety of kinematical models for rotating and expanding (or contracting) disks and for radial flow in cones. Extranuclear profiles of optically thin lines are predicted by integration along prespecified line of sights. The models are parameterized by radial power laws of line emissivity $\varepsilon=r^{b}$ and circular or radially directed velocity $w=r$.

The NLR is slightly extended in [OIII] along PA $50^{\circ} \pm 20^{\circ}$ and its largest kinematical gradient appears along $40^{\circ} \pm 20^{\circ}$ which reinforces Anderson's (1974) suggestion of a single rotating NLR disk. Despite a good match of the profile centroids single-disk models appear to be ruled out because they require turbulence widths exceeding twice the rotational velocity.

However, many profiles are either split, flat-topped or show shoulders. These line components show a spatial pattern which suggests that they arise in different dynamical entities (Ulrich 1973). In $\mathrm{H} \alpha$ the inward extension of the flat rotation curve of the galactic disk can be traced down to $R=2$ " by components with $\sim 1060 \mathrm{~km} \mathrm{~s}^{-1}$ in the $\mathrm{NE}$ and $\sim 940 \mathrm{~km} \mathrm{~s} \mathrm{~s}^{-1}$ in the SW. A conspicuous blueshifted component at $740 \pm 20 \mathrm{~km} \mathrm{~s}^{-1}$, present in high and low-excitation lines between PA $160^{\circ}$ and $330^{\circ}$, cannot reasonably be traced back to motion in the galactic disk. Its ubiquity over $\sim 600 \mathrm{pc}$ and the small 
velocity variation of the feature calls for a huge wall of material approaching us, probably the leading surface of a superbubble. Its far side appears to merge with the disk component. Evidence for an additional, less violent, bubble, stronger in [OIII] than in $\mathrm{H} \alpha$, with components at 980 and $1150 \mathrm{~km}$ $\mathrm{s}^{-1}$ exists in the NE. It ends beyond $R=4 "$ where [OIII] joins $\mathrm{H} \alpha$ in attaining normal galactic disk rotation. No component structure is seen in the ranges PA $90^{\circ}-160^{\circ}$ and $330^{\circ}-360^{\circ}$ which hence appear to lie outside the bubbles in the picture suggested here. The FWHM of the components (measured at $R=$ $4 "$, in order to minimize seeing effects) amount to $150-200 \mathrm{~km} \mathrm{~s}^{-1}$, corresponding to $\sigma=60-90 \mathrm{~km} \mathrm{~s}^{-1}$.

Assuming for the components $10 \%$ to $50 \%$ of the total $\mathrm{H} \beta$ lumininosity $\mathrm{L}_{\mathbf{H} \beta}(\mathrm{NLR})=21040$ erg $\mathrm{s}^{-1}$, Balzano's (1983) $\mathrm{L}_{\mathbf{H} \beta}-\sigma$ relation for starburst nuclei predicts $\sigma=25-40 \mathrm{~km} \mathrm{~s}^{-1}$, well below the observed velocity dispersion. Hence even the line components are broadened by something not common in starburst nuclei. Rotational broadening of the disk component can be ruled out by our models. Turbulence induced by differential rotation (Fleck 1981) would not suffice on the scales observed here. Our models show that radially directed outflow of the clouds inside a filled cone could produce a broad 740-component, but the scenario of a pressure-driven supershell is physically more likely. For this a quantitative theoretical prediction of the amount of turbulence is not available, but the observationally well documented bipolar flow in M82 (Bland and Tully 1988) shows typical FWHM $\sim 180 \mathrm{~km} \mathrm{~s}^{-1}$ of the lines emitted by the filaments from the surfaces of the bubbles, close to the values required here.

The hot cavity of a superbubble or wind-shocked clouds are expected to emit soft $X$-rays which are indeed observed from the SW NLR (Elvis et al., 1983) with a luminosity comparable to that from M82. The different geometry (face-on view versus edge-on view) might even explain the weak NL-polarisation in NGC 4.151 as compared to the strong $90^{\circ}$-scattering polarisation from a dust halo in M82. In addition, the hole in the SW disk invoked to explain the UV-radiation cone exciting a jet-like string (Schulz 1988) may be simply produced by the SW superbubble. This suggests that the superbubbles are related to the central radio jet because Unger et al. (1987) found a correlation between the directions of the radio axis and the extranuclear strings. Also the line width - radio power correlation (Whittle 1985) indicates a relation with the radio source.

Anderson, K.S. 1974, Ap. J. 189, 195

Balzano, V.A. 1983, Ap. J. 268, 602

Bland J., Tully, R.B. 1988, Nature 334, 43

Elvis, M., Briel, U.G., Henry, J.P. 1983, Ap. J. 268, 105

Fleck, R.C. 1981, Ap. J. 246, L151

Schulz, H. 1987, Astr. Ap. 178, 7

Schulz, H. 1988, Astr. Ap., in press

Ulrich, M.-H. 1973, Ap. J. 181, 51

Unger, S.W., Pedlar, A., Axon, J.A., Whittle, M., Meurs, E.J.A., Ward, M.J. 1987 M.N.R.A.S. 228, 671

Whittle, M. 1985, M.N.R.A.S. 213, 33 


\section{DISCUSSION}

WHITTLE Could you remind us of the position of the linear radio source and comment on whether the red/blue profile components could be explained in terms of a direct interaction between a jet and the interstellar matter, as implied for some other Seyfert galaxies.

SCHULZ The direction of the radio source differs from that of the extranuclear string and the implied UV radiation cone by about $20^{\circ}$. The SW blue/red components extend from PA $160^{\circ}$ to $33^{\circ}$ which I interpret to arise mainly in the turbulent shells of a super-bubble. Combining this with the statistical evidence by Unger et al., I suggest that the radio jet directly or indirectly gave rise to the super-bubble which distorted the galactic disk so that the UV-radiation cone could emerge. 